\title{
INFORME DE PARTICIPACIÓN EN REUNIÓN DEL CONSEJO EJECUTIVO DE LA ASOCIA- CIÓN LATINOAMERICANA DE FACULTADES Y ESCUELAS DE CONTADURIAA-ALAFEC
}

Dr. Ernesto Augusto Polar Falcón

\begin{abstract}
RESUMEN
Del 09 al 11 de Abril del 2003, se realizó la Reunión de Consejo Ejecutivo de la ALAFEC en la Universidad de La Habana-Cuba, con asistencia de los directivos, Decanos de Facultades y Escuelas de Contaduría de América Latina e invitados especiales. La Facultad de Ciencias Contables de la Universidad Nacional Mayor de San Marcos estuvo presente con la participación del Dr. Julio Vicente Flores Konja, en su condición de Decano y Miembro del Consejo Ejecutivo y el Dr. Ernesto Augusto Polar Falcón en su condición de Director del Instituto de Investigación Contable de Ciencias Financieras y Contables; así como expositor del trabajo titulado "Desarrollo y Alcances de la Investigación en la UNMSM y en la Facultad de Ciencias Contables de la UNMSM". Se presenta un resumen de dicha exposición y se transcribe el importante documento titulado: "Creación de la Red Internacional de la ALAFEC».
\end{abstract}

\section{ANTECEDENTES}

1. La Asociación Latinoamericana de Facultades y Escuelas de Contaduría Pública -ALAFECprecisa en el Artículo $\mathrm{I}^{\circ}$ de su Estatuto, que es un organismo internacional, interuniversitario y regional, que tiene por finalidad principal propender a la cooperación y entendimiento de sus asociados, con el objetivo de unificar y elevar el nivel de enseñanza, investigación y capacitación técnica y científica de los contadores y auditores con vistas al desarrollo de América Latina.

2. El 17 de junio de 1982 se constituye la ALAFEC como Asociación, durante la II Conferencia de Facultades, Escuelas, Departamentos y Programas de Contaduria Pública de América Latina, realizada en la Universidad Autónoma de Santo Domingo, República Dominicana, del 13 al 17 de junio de 1982.

* Profesor Principal de la Facultad de Ciencias Contables y Director del Instituto de Investigaciones de Ciencias Financieras y Contables.

Miembro del Comité Ejecutivo del Consejo Superior de Investigaciones-UNMSM, Representante del Ârea Económico-Empresarial. 
3. ALAFEC, recibe el apoyo de la Unión de Universidades de América Latina-UDUAL, con sede en México, representada por la Universidad Nacional Autónoma de México. La Universidad Nacional Mayor de San Marcos es miembro de la UDUAL, cuyo apoyo abarca a Asociaciones y

$\begin{array}{lllr}\text { Área I } & \text { - Región Andina } & 54 \\ \text { Área II } & \text { - Región México y Caribe } & 121 \\ \text { Área III } & \text { - Región Centroamérica } & 5 \\ \text { Área IV } & \text { - Región Cono Sur } & & 24 \\ & & & \text { Total }\end{array}$

Organizaciones de Derecho, Economía, Enfermeria Contaduria, Medicina, Odontologia, Psicologia, etcetéra.

4. ALAFEC agrupa a 204 instituciones de educación profesional, distribuidas en cuatro Áreas como sigue:

\section{$N^{\circ}$ de Instituciones}

\section{5}

24
5. En los primeros meses del año 2002, ALAFEC invita a la Universidad Nacional Mayor de San Marcos a liderar activamente su participación como representante de Perú ante la ALAFEC, por el gran prestigio internacional que tiene. La invitación la recibe el Dr. Julio Vicente Flores Konja, Decano de la Facultad de Ciencias Contables de la Universidad Nacional Mayor de San Marcos, para integrarse como Miembro del Consejo Ejecutivo de ALAFEC, quien asiste a dos reuniones del Consejo Ejecutivo de ALAFEC, realizadas en México-Facultad de Contaduría y Administración de la Universidad Nacional Autónoma de México, el 18 y 19 de abril del 2002; y en Chile-Facultad de Contabilidad y Auditoría de la Universidad de Santiago de Chile, el 17 y 18 de octubre del 2002.

6. En la Reunión del Consejo Ejecutivo de México, el Dr. Julio Vicente Flores Konja se integra a la Comisión de Trabajo encargada de crear la Red Internacional de Investigadores.

7. Para la Reunión del Consejo Ejecutivo, realizado en CubaFacultad de Contabilidad y Finanzas de la Universidad de La Habana, del 09 al 11 de abril del 2003 asisten el Dr. Julio Vicente Flores Konja, Decano de la Facultad de Ciencias Contables y Miembro del Consejo Ejecutivo de ALAFEC y el Dr. Ernesto Augusto Polar Falcón, Director de Investigaciones de la Facultad de Ciencias Contables, Miembro del Comité Ejecutivo del Consejo Superior de Investigaciones y expositor del trabajo preparado con el título de "Desarrollo y Alcances de la Investigación en la UNMSM y en la Facultad de Ciencias Contables", como aporte a la implementación del Directorio Nacional del Perú en la Red Internacional de Investigadores en Contaduria de ALAFEC. 
A. EXPOSICIÓN: "DESARROLLO $Y$ ALCANCES DE LA INVESTIGACIÓN EN LA UNMSM Y EN LA FACULTAD DE CIENCIAS CONTABLES"

1. En la Primera Sesión de Trabajo del jueves 10 de abril del 2003, que se inició a las diez horas y terminó a las dieciséis horas, se discutieron los 11 puntos de la Orden del Día.

2. En el punto 11, el tema fue: «Plan de Acción para la Integración del Directorio Internacional de Investigadores ALAFEC" a cargo de maestro Jorge Díaz Szalay y del Le. Leonel Sebastián.

3. En el subtema: "Avances de la Investigación Universitaria», el maestro Arturo Díaz Alonso, Presidente de ALAFEC, concedió el uso de la palabra al Dr. Ernesto Augusto Polar Falcón, para efectuar la exposición audiovisual con el apoyo de imágenes en Power Point de la Conferencia titulada: "Desarrollo y Alcances de la Investigación en la UNMSM y en la Facultad de Ciencias Contables", cuyo resumen es el siguiente:

3.1. Se presentó el saludo de la UNMSM a los distinguidos asistentes a la Reunión del Consejo Ejecutivo, formado por los Decanos de las Facultades y Escuelas de Contaduría de América Latina, agradeciendo la oportunidad brindada para transmitir los conocimientos en el tema de la investigación universitaria, incrementados en los últimos años, al ejercer los cargos actuales en la UNMSM, de
Director del Instituto de Investigación de Ciencias Financieras y Contables de la Facultad de Ciencias Contables y Miembro del Comité Ejecutivo del Consejo Superior de Investigaciones.

3.2. La primera parte de la exposición estuvo centrada en mostrar el contenido del documento titulado "Políticas y Estrategias-Quinquenio 2002-2006" que ha preparado el Consejo Superior de Investigaciones de la UNMSM, con el apoyo de los miembros del Comité Ejecutivo.

3.3. Se presentaron las áreas de Visión, Misión y Valores que propicia el Consejo Superior de Investigaciones, cuyas siglas más conocidas son las iniciales CSI; luego se expuso específicamente el tema principal relacionado con las "Políticas y Estrategias" mostrando la "Presentación", los "Antecedentes" y el "Análisis Situacional" del CSI; para luego presentar los siguientes "Lineamientos" de sus políticas y estrategias para el Quinquenio 2002-2006:

Lineamientos

A. Políticas y Alianzas

Objetivos: líneas de Investigación Convenios Específicos Publicación

B.Política de Formación de Investigadores

Objetivos: Procesos de Acreditación

Nuevos Investigadores

Recursos Humanos 
C. Política de Recursos Económicos

Objetivos: Proyectos

Gestionadores

Remuneración para

Investigadores

Estructuras y Equipos

D. Política de Nueva estructura del CSI

Objetivos: Administración

Presupuesto

Investigación

Multidisciplinaria

3.4. A continuación se expuso el tema de las "Fuentes de Financiamiento" que propone el CSI, con el siguiente contenido:

A. Universidad Nacional Mayor de San Marcos

Política: Tesoro Público

Recursos Propios

B. Recursos Nacionales

Política: Donaciones en el Marco Tributario.

Convenios con

In s t it u c iones

Estatales.

Convenios con

Instituciones $\sin$ fines

de lucro.

Convenios con

Universidades

Peruanas.

C. Recursos del Exterior

Políticas: Convenios con

Entidades del

Exterior.

Convenios para

Recepción de

Bienes y Servicios.

Convenios con

Universidades

Extranjeras.
3.5. Se agregó en la exposición, el proceso de investigación que anualmente aplica la UNMSM indicando:

- Formación de 5 áreas de Investigación:

- Ciencias Básicas

- Ciencias de la Salud

- Ingenierías

- Económico Empresariales

- Humanidades

- Los 31 institutos y centros de investigación

- Las lineas de investigación

- La Infraestructura Anual de 250 Estudios de Investigación distribuidos en las 5 áreas de investigación

- El uso de formularios y procedimientos estatutarios anuales aplicados exclusivamente en la investigación universitaria

3.6. En la segunda parte de la exposición se presentaron los cuadros de Estudios de Investigación que realiza $y$ ha realizado la Facultad de Ciencias Contables de la UNMSM en los últimos seis años como sigue:

$$
\begin{aligned}
& \text { Año: } 2003 \text { - } 9 \text { Estudios } \\
& 2002 \text { - } 12 \text { Estudios } \\
& 2001 \text { - } 8 \text { Estudios } \\
& 2000 \text { - } 9 \text { Estudios } \\
& 1999 \text { - } 7 \text { Estudios } \\
& 1998 \text { - } 6 \text { Estudios }
\end{aligned}
$$

3.7. Se incluyó el proceso de calificación de los Estudios 
de Investigación, que aplica la Facultad de Ciencias Contables, mostrando el trabajo de un Jurado Examinador y el Cuadro de Número y Puntaje de Publicaciones Científicas que se usan para identificar a los docentes Responsables.

3.8. Las reacciones al término de la exposición, fueron variadas. Los decanos de Ecuador y Bolivia manifestaron que recién iniciaban actividades de investigación en sus Facultades y se mostraron sorprendidos de los avances de investigación universitaria en el Perú. Otros decanos se mostraron interesados en las Políticas y Estrategias del Plan Guinquenal 2002-2006.

3.9. Las discusiones derivaron a la necesidad de aplicar las políticas para obtener el financiamiento de la investigación y qué se deberia tomar en cuenta para la formación del Directorio Nacional en cada país latinoamericano, en la formación de la Red Internacional de Investigadores en Contaduría de ALAFEC.

4. Se propuso que los actuales Investigadores de las Facultades y Escuelas de Contaduría de América Latina sean los iniciadores de los Directorios Nacionales de cada país. De aprobarse esta propuesta los docentes responsables de los Estudios de Investigación 2003, de la Facultad de Ciencias Contables de la UNMSM serian nombrados Investigadores $\mathrm{y}$ formarian el Directorio Nacional del Perú en la Red Internacional de Investigadores en Contaduria de ALAFEC.

5. Como corolario de esta exposición, los decanos de Ecuador, Bolivia y Nicaragua, mostraron interés en firmar Convenios con la UNMSM para recibir la asesoria requerida en el fomento de la investigación universitaria en sus respectivas Facultades y Escuelas de Contaduría.

\section{B. RED INTERNACIONAL DE INVESTIGADORES DE ALAFEC (RII ALAFEC)}

1. Se presentó en la Reunión de Consejo Ejecutivo de ALAFEC el documento titulado "CREACIÓN DE LA RED INTERNACIONAL DE INVESTIGADORES DE LA ALAFEC (RIIALAFEC)" que se incluye en el presente Informe como ANEXO A.

2. Este documento consta de los siguientes títulos:

- Antecedentes

- Objetivos

- Acciones Propuestas

2.1. Elaboración de Directorio Latinoamericano de Investigadores ALAFEC

- Los puntos tratados más importantes son:

- El Directorio Latinoamericano deberá constituirse con los Directorios Nacionales.

- Convocatoria General para Latinoamérica y que cada país lance su convocatoria nacional. 
- Información de cada investigador

- Uniformar criterios referente a:

- Áreas de Investigación

- Líneas de Investigación

2.2. Creación de un Órgano Coordinador de la Red

- Funciones Transitorias

- Funciones Permanentes

3. Se presentó también el formulario titulado "Directorio Nacional de Investigadores" de uso actual en la Asociación Nacional de Facultades y Escuelas de Contaduria y Administración-ANFECA, que se propone aplicar, el cual contiene los siguientes títulos:

- Información Personal

- Información Laboral

- Formación Académica

- Actividades de Investigación:

- Áreas de Conocimiento

- Líneas de Investigación

- Trabajos de Investigación Publicados

- Experiencia Docente

4. Se propuso que las Facultades y Escuelas que ya tienen organizaciones propias de docentes investigadores constituyan el respectivo Directorio Nacional.

5. A la recepción de los acuerdos aprobados de la presente Reunión del Consejo Ejecutivo de ALAFEC, la Facultad de
Ciencias Contables de la UNMSM podría formar de inmediato el Directorio Nacional del Perú de la Red de Investigadores de ALAFEC (RIIALAFEC), con el equipo docentes responsables de los estudios de investigación incluyendo a los Docentes Miembros A, Miembros B y Colaboradores.

\section{ANEXO A CREACIÓN DE LA RED INTERNACIONAL DE INVESTIGADORES DE LA ALAFEC (RIIALAFEC)}

(Versión aprobada en la reunión del Consejo Ejecutivo de ALAFEC celebrada en Santiago de Chile)

\section{ANTECEDENTES}

Consciente de que es impostergable que se dé un impulso vigoroso a la investigación en contaduría en las universidades latinoamericanas con el fin de generar conocimientos en lugar de sólo transmitirlo acríticamente, la ALAFEC ha decidido crear su Red Internacional de Investigadores en Contaduría (RIIALAFEC). Dicha Red se funda en la premisa de que el avance del conocimiento en cualquier disciplina históricamente se ha dado y se seguirá dando sólo gracias al diálogo entre los investigadores de la misma, el cual comprende no sólo el que establecen oralmente con sus colegas o pares, sino también y principalmente el que entablan a través de la palabra escrita con pensadores presentes, pasados e incluso futuros. La necesidad de dicho proceso, que implica el conocimiento y anảlisis del pensamiento de otros, queda claramente ejemplificada en el multicitado pensamiento de Newton "Si he visto más allá, es por haberme colocado en los hombros de gigantes». 
Hasta hace poco tiempo, unas dos décadas, dicho diálogo se realizaba cotidianamente a través de lo dicho por los investigadores en libros, revistas especializadas y, en algunos casos, en contactos personales; ocasionalmente se llevaba a cabo, además, por medio de eventos académicos diversos (congresos, simposia, conferencias, etc.) Todos estos medios que hoy en día siguen y continúan siendo por largo tiempo empleados para el desarrollo del conocimiento científico y técnico han recibido un impulso importante de los nuevos medios electrónicos que facilitan y aceleran el intercambio de pensamiento entre los investigadores. Estos medios electrónicos sin duda habrán de ayudarnos también en la Red Internacional que estamos creando, pero de ninguna manera substituyendo a los medios tradicionales. sino sólo apoyándolos como vehículos de comunicación.

\section{OBJETIVOS}

Las redes de investigadores coadyuvan a la investigación facilitando el intercambio de ideas entre aquéllos y la realización de proyectos de investigación conjuntos. En el caso concreto de la ALAFEC, su Red Internacional de Investigadores en Contaduría busca alcanzar los siguientes objetivos:

Impulsar la investigación tanto básica como aplicada en contaduría a través de:

Facilitar y multiplicar el intercambio académico de pensamientos y hallazgos entre los investigadores latinoamericanos, principalmente por medio de artículos, ensayos y sugerencias bibliográficas, asi como de criticas y consultas mutuas.

- Promover la realización de proyectos de investigación conjuntos.

Promover la participación de los investigadores latinoamericanos en eventos académicos de los diferentes paises, así como el intercambio de investigadores, estudios y materiales de investigación.

\section{ACCIONES INICIALES PROPUESTAS}

1. Elaboración del Directorio Latinoamericano de Investigadores ALAFEC

Para el establecimiento de la red es fundamental la elaboración de un Directorio Latinoamericano de Investigadores ALAFEC, el cual se deberá ir alimentando de directorios nacionales que ya están o que elaboraremos en cada uno de nuestros paises. De hecho, dicho Directorio será el instrumento central o corazón de la Red.

Para la elaboración del Directorio proponemos que se lance una convocatoria general para Latinoamérica y que en cada pais se lance a su vez una convocatoria nacional para la conformación de los directorios correspondientes.

Para que sean instrumentos prácticos y de relativamente fácil actualización, tanto el directorio latinoamericano como los directorios nacionales deberán contener poca información pero muy pertinente. Si ésta es complicada el directorio y la red se volverán pronto obsoletos. Se estima que la información necesaria acerca de cada investigador es la siguiente:

- Nombre y demás datos de identificación del investigador (domicilio, dirección de correo electrónico, teléfono [s]).

- Institución en que labora.

- Área(s) de investigación.

- Línea(s) de investigación que trabaja.

- Títulos de los trabajos que ha realizado en la misma línea de investigación y referencias de su publicación, en su caso. 
Hasta hace poco tiempo, unas dos décadas, dicho diálogo se realizaba cotidianamente a través de lo dicho por los investigadores en libros, revistas especializadas y, en algunos casos, en contactos personales; ocasionalmente se llevaba a cabo, además, por medio de eventos académicos diversos (congresos, simposia, conferencias, etc.) Todos estos medios que hoy en dia siguen y continúan siendo por largo tiempo empleados para el desarrollo del conocimiento científico y técnico han recibido un impulso importante de los nuevos medios electrónicos que facilitan y aceleran el intercambio de pensamiento entre los investigadores. Estos medios electrónicos sin duda habrán de ayudarnos también en la Red Internacional que estamos creando, pero de ninguna manera substituyendo a los medios tradicionales, sino sólo apoyándolos como vehículos de comunicación.

\section{OBJETIVOS}

Las redes de investigadores coadyuvan a la investigación facilitando el intercambio de ideas entre aquéllos y la realización de proyectos de investigación conjuntos. En el caso concreto de la ALAFEC, su Red Internacional de Investigadores en Contaduría busca alcanzar los siguientes objetivos:

Impulsar la investigación tanto básica como aplicada en contaduría a través de:

Facilitar y multiplicar el intercambio académico de pensamientos y hallazgos entre los investigadores latinoamericanos, principalmente por medio de artículos, ensayos y sugerencias bibliográficas, así como de criticas y consultas mutuas.

- Promover la realización de proyectos de investigación conjuntos.

Promover la participación de los investigadores latinoamericanos en eventos académicos de los diferentes países, asi como el intercambio de investigadores, estudios y materiales de investigación.

\section{ACCIONES INICIALES PROPUESTAS}

1. Elaboración del Directorio Latinoamericano de Investigadores ALAFEC

Para el establecimiento de la red es fundamental la elaboración de un Directorio Latinoamericano de Investigadores ALAFEC, el cual se deberá ir alimentando de directorios nacionales que ya están o que elaboraremos en cada uno de nuestros países. De hecho, dicho Directorio será el instrumento central o corazón de la Red.

Para la elaboración del Directorio proponemos que se lance una convocatoria general para Latinoamérica y que en cada país se lance a su vez una convocatoria nacional para la conformación de los directorios correspondientes.

Para que sean instrumentos prácticos y de relativamente fácil actualización, tanto el directorio latinoamericano como los directorios nacionales deberán contener poca información pero muy pertinente. Si ésta es complicada el directorio y la red se volverán pronto obsoletos. Se estima que la información necesaria acerca de cada investigador es la siguiente:

- Nombre y demás datos de identificación del investigador (domicilio, dirección de correo electrónico, teléfono [s]).

- Institución en que labora.

- Área(s) de investigación.

- Linea(s) de investigación que trabaja.

- Títulos de los trabajos que ha realizado en la misma línea de investigación y referencias de su publicación, en su caso. 
- Proyecto de investigación actual y proyecto(s) futuro(s)

- Currículo resumido.

Con el objetivo de uniformar los criterios respecto a lo que deberá entenderse por área y línea de investigación, lo cual es de suma importancia, al lanzar las convocatorias se incluirán las siguientes definiciones:

Área de investigación. Rama o campo de conocimiento de la contaduría sobre el cual se pretende realizar investigación, y que comprende una o varias líneas de investigación afines. Para el caso de la carrera de Contaduria, se han definido las siguientes 9 áreas de investigación:

I. Área Básica

\section{Finanzas}

2. Contabilidad

3. Auditoría

4. Costos

5. Contribuciones y Tributación (Fiscal)

6. Control

\section{II. Áreas Complementarias}

\section{7. Ética}

8. Formación Profesional

\section{Gestión Ambiental}

Línea de investigación. Tema o problemática específica de investigación de un área dentro de la cual se pueden inscribir una infinidad de proyectos de investigación individuales o colectivos. Su fin primordial es el de señalar y delimitar a muy grandes rasgos, mediante un simple titulo, un tema o problemática de interés de un investigador y/o un centro de investigación. Por ejemplo: métodos de valuación de empresas, como línea del área finanzas; principios de contabilidad nacionales e internacionales, como linea de área contabilidad; costos $\mathrm{ABC}$ como línea del área de costos; teoría del portafolios de inversión, como línea del área de finanzas; curricula y desarrollo académico, como línea del área formación profesional.

\section{Creación de un Órgano Coordinador de la Red}

Se propone la creación de una Coordinación de la Red Internacional de Investigadores en Contaduria, ALAFEC, con las siguientes funciones principales:

\section{Funciones transitorias}

Continuación de la elaboración del presente proyecto y coordinación de las actividades que deban efectuarse para convertirlo en realidad una vez que sea aprobado.

Elaboración de una propuesta de página de Internet de la Red y establecimiento de una dirección de correo electrónico de la misma.

Apertura de la página de Internet de la Red una vez que haya sido aprobada.

Elaboración de la propuesta de convocatoria para conformar el Directorio Latinoamericano de Investigadores en Contaduria.

Publicación de la convocatoria para la conformación del Directorio citado en diversos medios, incluyendo la propia página de Internet de la Red.

- Depuración y ordenación de la información obtenida en respuesta a la convocatoria lanzada y publicación de la misma como Directorio Latinoamericano de Investigadores en contaduria.

\section{Funciones permanentes}

Coordinación de todas las actividades tendientes a la operación de la Red, asi como a su actualización y mejora

Promoción para lograr que la Red se amplie mediante la inscripción de nuevos investigadores. 


\section{CONCLUSIONES}

1. La exposición del tema "Desarrollo y Alcances de la Investigación en la UNMSM y en la Facultad de Ciencias Contables" tuvo una favorable acogida por parte de los Decanos de Facultades y Escuelas de Contaduria de América Latina, que asistieron a la Reunión del Consejo Ejecutivo de ALAFEC, por cuanto se mostraron gratamente sorprendidos de que la UNMSM tuviera en perspectiva un plan quinquenal de políticas y estrategias sobre investigación universitaria; una organización adecuada que lidera el Consejo Superior de Investigación, coordinando con las Unidades e Institutos de Investigaciones de las 20 Facultades, la realización de los Estudios de Investigación en sus diferentes modalidades, la publicación de revistas de investigación y el desarrollo de los talleres anuales de Investigación; una excelente distribución del control de la investigación universitaria en el marco de las 5 áreas de investigación, en las que se han alineado las 20 facultades de la UNMSM y en el notable esfuerzo que se realiza para colocar las publicaciones de sus revistas especializadas en los registros indexados internacionales.

2. En la misma exposición, el tema de las actividades de investigación que realiza la Facultad de Ciencias Contables de la UNMSM tuvo favorables comentarios de parte de los Decanos de Facultades y Escuelas de Contaduria, afirmando algunos de ellos que en sus Facultades aún no habian alcanzado un nivel de avance como el que presentaba la Facultad de Ciencias Contables de la UNMSM, por la variedad de los temas de investigación que realizan en el presente año 2003 y los realizados en años anteriores; la disciplina del proceso de selección a cargo de un jurado calificador y el uso correcto del Formulario de Estudios de Investigación que aplicaban en cumplimiento de las normas reglamentarias establecidas para la investigación universitaria.

3. La recomendación dada en la Reunión del Consejo Ejecutivo de ALAFEC para la formación del Directorio Nacional del Perú en la Red Internacional de Investigadores en Contaduria de la ALAFEC con los actuales docentes investigadores de cada Facultad o Escuela de Contaduria en toda la América Latina es una medida acertada, cuya confirmación oficial permitirá que, en el caso del Perú, la Facultad de Ciencias Contables de la UNMSM efectúe la inscripción inmediata de sus Docentes Responsables, Miembros A y B y Colaboradores de Estudios de Investigación 2003, en el Directorio Nacional del Perú para formar parte de la Red Internacional de Investigadores en Contaduria de ALAFEC.

4. Las inscripciones de docentes investigadores de Facultades y Escuelas de Contaduría de Universidades Peruanas, que propiciaria la Facultad de Ciencias Contables de la UNMSM, con un corto plazo, en su condición de representante de Perú en el Grupo Andino de 
ALAFEC, ampliará el Directorio Nacional del Perú en la Red Internacional de Investigadores en Contaduría de la ALAFEC, reforzando notablemente la presencia del Perú en las actividades de investigación universitaria, a nivel nacional y latinoamericano, haciendo realidad los proyectos de desarrollo de la investigación que propicia la Asociación Latinoamericana de Facultades y Escuelas de Contaduria-ALAFEC. 